\title{
TEMPERATURE STUDIES OF OPTICAL BIREFRINGENCE IN MOLECULAR LIQUIDS. I. LIQUIDS WITH LINEAR TEMPERATURE DEPENDENCE
}

\author{
Z. Blaszczak and A. DoIari \\ Institute of Physics, A. Mickiewicz University, Grunwaldzka 6, 60-780 Poznań, Poland \\ (Received December 6, 1991; in final form April 10, 1992)
}

\begin{abstract}
We report the first systematic study of the temperature dependence of optically induced birefringence in benzene, toluene, nitrobenzene, chlorobenzene, 2-bromoanisole, 2-methylbenzaldehyde, 3-methylbenzaldehyde, 3-chlorobenzaldehyde and cinnamaldehyde. The optical Kerr constants, relative to benzene, are measured in a broad temperature range. Refractive indices and densities of the liquids for the temperatures studied were also determined. For the above liquids the $T^{-1}$ temperature dependences of the optical Kerr constants have been found. From our measurements, the molar Kerr constants and the effective molecular anisotropies were calculated as functions of temperature. The mean values of the second-order optical hyperpolarizability have also been determined.
\end{abstract}

PACS numbers: 35.20.My, 42.60.He, 78.20.Dj

\section{Introduction}

Nonlinear optical effects in liquids are shaped not only by the optical properties of isolated atoms (molecules) but moreover by their electric properties and mutual interactions as well. Hence, optical birefringence induced by strong laser light, also referred to as the optical Kerr effect (OKE), is a source of highly relevant information concerning the structure, electric and optical properties of atoms and molecules including their interactions in dense media. OKE measurements yield the optical Kerr constant $B_{0}\left(\lambda_{\mathrm{a}}, \lambda_{\mathrm{i}}\right)$, which characterizes macroscopic properties of the medium. If carried out versus temperature, OKE measurements provide information on the local structure of the medium and permit the calculation of the nonlinear optical polarizability and effective optical anisotropy of molecules making use of the molecular-statistical theory of OKE [1]. According to the latter, the molar optical Kerr constant $B_{\mathrm{m}}\left(\lambda_{\mathrm{a}}, \lambda_{\mathrm{i}}\right)$, dependent on molecular mechanisms 
leading to induced birefringence, is related with the microscopic properties of the system. $B_{\mathrm{m}}\left(\lambda_{\mathrm{a}}, \lambda_{\mathrm{i}}\right)$ can be expressed as the sum of two components

$$
B_{\mathrm{m}}\left(\lambda_{\mathrm{a}}, \lambda_{\mathrm{i}}\right)=B_{\mathrm{m}}^{\mathrm{NL}}\left(\lambda_{\mathrm{a}}, \lambda_{\mathrm{i}}\right)+B_{\mathrm{m}}^{\mathrm{RT}}\left(\lambda_{\mathrm{a}}, \lambda_{\mathrm{i}}\right)
$$

of which the first one is temperature-independent and determines the contribution to OKE from nonlinear deformation of the electron shell of the atoms (molecules) in the optical field (Voigt's effect), whereas the second component, which is a function of temperature, determines the contribution from molecular reorientation in the optical field (Langevin effect) and from molecular-statistical processes. The Kerr constant $B_{\mathrm{m}}^{\mathrm{NL}}\left(\lambda_{\mathrm{a}}, \lambda_{\mathrm{i}}\right)$ depends on the nonlinear properties of the atoms (molecules) described by the tensor of second-order hyperpolarizability $c_{\alpha \beta \gamma \delta}$ and, moreover, on the intermolecular interactions. It can be expressed in a general form [2] as

$$
B_{\mathrm{m}}^{\mathrm{NL}}\left(\lambda_{\mathrm{a}}, \lambda_{\mathrm{i}}\right)={ }_{0} B_{\mathrm{m}}^{\mathrm{NL}}+{ }_{\mathrm{inter}} B_{\mathrm{m}}^{\mathrm{NL}},
$$

where the first term describes the Voigt's effect in the case when there are no molecular interactions in the medium, and the second term regards all types of molecular interactions. The interaction contribution to $B_{\mathrm{m}}^{\mathrm{NL}}$ varies from one liquid to another and can range from several up to tens of \% [3] in the case of liquids composed of strongly interacting molecules. For liquids the molecules of which have low permanent electric moments and weak optical anisotropy the influence of molecular interactions on $B_{\mathrm{m}}^{\mathrm{NL}}$ can be neglected in the first approximation (the second term in Eq. (2) vanishes). $B_{\mathrm{m}}^{\mathrm{NL}}$ then takes the form [4]

$$
B_{\mathrm{m}}^{\mathrm{NL}}\left(\lambda_{\mathrm{a}}, \lambda_{\mathrm{i}}\right)=\frac{4}{9} \pi N_{\mathrm{A}} c,
$$

with $N_{\mathrm{A}}$ the Avogadro's number and $c$ the mean value of the tensor $c_{\alpha \beta \gamma \delta}$ defined by the relation $c=\frac{1}{5} c_{\alpha \alpha \beta \beta}$.

The constant $B_{\mathrm{m}}^{\mathrm{RT}}$ can be expressed as follows [4]:

$$
B_{\mathrm{m}}^{\mathrm{RT}}\left(\lambda_{\mathrm{a}}, \lambda_{\mathrm{i}}\right)=\frac{4 \pi N_{\mathrm{A}}}{45 k T} \Gamma^{2}\left(\lambda_{\mathrm{a}}, \lambda_{\mathrm{i}}\right),
$$

where $\Gamma$ is the effective optical anisotropy characterizing the molecular optical anisotropy $\gamma$ of the molecule in the medium. For rarefied gases only, one can assume the effective anisotropy as equal to the anisotropy of the isolated molecule. However, the influence of its nearest neighbourhood on the optical properties of a molecule increases with growing density of the medium and becomes considerable in liquids. This leads, chiefly by way of multipolar interactions significantly dependent on the structure of the molecules, to the appearance of regions of short-range ordering in the liquid and to the formation of momentary molecular assemblages. It should be remembered that in dense media the molar Kerr constant $B_{\mathrm{m}}\left(\lambda_{\mathrm{a}}, \lambda_{\mathrm{i}}\right)$ and the experimentally determined optical Kerr constant $B_{0}\left(\lambda_{\mathrm{a}}, \lambda_{\mathrm{i}}\right)$ are interrelated as follows [2]:

$$
B_{0}\left(\lambda_{\mathrm{a}}, \lambda_{\mathrm{i}}\right)=\frac{3}{2 n_{\mathrm{a}} \lambda_{\mathrm{a}} V_{\mathrm{M}}}\left(\frac{n_{\mathrm{a}}^{2}+2}{3}\right)^{2}\left(\frac{n_{i}^{2}+2}{3}\right)^{2} B_{\mathrm{m}}\left(\lambda_{\mathrm{a}}, \lambda_{\mathrm{i}}\right)
$$

permitting the calculation of the molar Kerr constants from the experimental results. Above, $n_{\mathrm{a}}$ and $n_{\mathrm{i}}$ are the refractive indices for the inducing light beam and analysing beam, respectively, and $V_{M}$ is the molar volume of the compound 
under investigation. Taking into consideration the temperature dependence of the molar Kerr constants, Eq. (1) becomes

$$
B_{\mathrm{m}}\left(\lambda_{\mathrm{a}}, \lambda_{\mathrm{i}}\right)=a+b T^{-1},
$$

where $a=(4 \pi / 9) N_{\mathrm{A}} c$ and $b=\left(4 \pi N_{\mathrm{A}} / 45 k\right) \Gamma^{2}\left(\lambda_{\mathrm{a}}, \lambda_{\mathrm{i}}\right)$. Studying $B_{\mathrm{m}}$ versus the inverse temperature $T^{-1}$ one determines the constants $a$ and $b$ and thus the values of the molar Kerr constants, hence the values of $c$ and $\Gamma^{2}$ can be derived.

As follows from the results of our studies, the temperature behaviour of the optical Kerr constants of certain molecular liquids reveals deviations from linearity in the low- or high-temperature part of the temperature range considered. This fact may provide additional information about the local structure of a given liquid. Therefore we decided to go into this problem in more detail and the investigation of the liquids showing nonlinear temperature dependence of optical birefringence and an attempt at its interpretation will be presented in Part II of this work.

\section{Experimental}

We applied the OKE setup designed and constructed in our Institute. OKE was induced with the beam of a ruby laser $\left(\lambda_{\mathrm{i}}=694.3 \mathrm{~nm}\right)$ generating nanosecond pulses $\left(\tau_{\text {pulse }}=10 \mathrm{~ns}\right.$, power $\left.20 \mathrm{MW}\right)$ and analysed with that of a continuously operating argon laser beam $\left(\lambda_{\mathrm{a}}=488 \mathrm{~nm}\right.$, of about $100 \mathrm{~mW}$ in power). For the principle and details of our setup we refer to our earlier papers $[7,8]$. Especially for the present investigation, it was supplemented with a thermostated Kerr cell and an electronic temperature-stabilizing device. The Kerr cell consisted of a $1 \mathrm{~cm}$ long, closed optical quartz cuvette placed within a metal shield containing heaters and a platinum resistor coupled to the temperature control device. The measuring and temperature stabilisation setup enabled us to vary the temperature of the liquids from ambient up to $300^{\circ} \mathrm{C}$ with an accuracy of $\pm 0.2^{\circ} \mathrm{C}$. For the present study we chose molecular liquids, where the phenomenon of induced birefringence was expected to be pronounced with regard to the presence of unsaturated bonds [1]. The liquids were of spectral purity, from Merck, Darmstadt, and after drying were introduced into the optical cuvette directly through a millipore filter.

\section{Results}

OKE measurements were performed for benzene as reference liquid and for the following molecular liquids: toluene, nitrobenzene, chlorobenzene, 2-bromoanisole, 2-methylbenzaldehyde, 3-methylbenzaldehyde, 3-chlorobenzaldehyde and cinnamaldehyde. For benzene and the first three above-mentioned liquids the temperature dependence of OKE was also studied by other researchers [5, 6]. For the rest of liquids these are the first OKE measurements and its temperature dependence. First, at room temperature, we determined their optical Kerr constants $B_{\text {rel }}$ relative to benzene. Next, we measured OKE as a function of temperature in the interval $T_{\text {room }}-T^{*}$, where $T^{*}=\left(T_{\text {boil }}-T_{\text {room }}\right) \times 85 \%$. Here $T_{\text {room }}$ is the ambient temperature, whereas $T_{\text {boil }}$ is the boiling temperature of the liquid. Setting the upper limit to the applied temperatures was a consequence of the fact that when 
approaching the boiling point of the liquid, significant optical inhomogeneities appeared in the studied medium which was the source of considerable inaccuracies of measurements. Therefore the value of $B_{\text {rel }}$ is for each temperature point the statistical mean of twenty to thirty measurements calculated by the least-squares method. The calculated error in determination of the values of $B_{\text {rel }}$ did not exceed $\pm 5 \%$.

With the well-known optical Kerr constant of benzene available [9], $B_{0}=$ $63.2 \cdot 10^{-9}[\mathrm{cgs}] \cdot\left(70.2 \times 10^{-16} \mathrm{~m} \cdot \mathrm{V}^{-2}\right)$, we calculated $B_{0}$ for the liquids $\left(B_{0}^{\text {liq }}=B_{\mathrm{rel}}^{\text {liq }} \times\right.$ $\left.B_{0}^{\text {benzene }}\right)$. In order to calculate the molar Kerr constants from Eq. (4) for a given temperature we need the densities $\rho$ of the liquids as well as their refraction indeces at the same temperatures involved. We determined these quantities experimentally, measuring $\rho$ for individual points throughout the entire range of temperatures $T_{\text {room }}-T^{*}$ and measuring the refraction indices with a Pulfrich refractometer only up to $50^{\circ} \mathrm{C}$ with regard to thermal limitations on the prism and calculating the indices $n_{\mathrm{a}}$ and $n_{\mathrm{i}}$ upwards of $50^{\circ} \mathrm{C}$ analytically from the linear relation of the type $n=a_{n}+b_{n} T$, where $T$ is temperature in Kelvin scale. The error due to divergences from linearity in $n(T)$ was assessed as several \% at the most. The obtained values of $a_{n}$ and $b_{n}$ are listed in Table A (Appendix).

Our measurements of $\rho$ versus $T$ for different temperatures led also to the linear dependence of the type $\rho=a_{\rho}+b_{\rho} T$, where $T$ is in Kelvin scale. The error due to divergences from linearity in $\rho(T)$ in the temperature range $10-50^{\circ} \mathrm{C}$, did not exceed $2 \%$. The calculated values of $a_{\rho}$ and $b_{\rho}$ are given in Table B (Appendix).

The results of our temperature measurements and calculations for the liquids studied are summarized in Table I.

\section{TABLE I}

The values of the refractive indices, densities, and Kerr constants $B_{\mathrm{rel}}, B_{0}$, and $B_{\mathrm{m}}$ of the liquids in their dependence on temperature $\left(\Delta T= \pm 0.2 \mathrm{~K}, \Delta n= \pm 0.0001, \Delta \rho= \pm 0.05 \mathrm{~kg} \cdot \mathrm{m}^{-3}, \Delta B_{\text {rel }}=\right.$ $\left.\Delta B_{0}=\Delta B_{\mathrm{m}}= \pm 5 \%\right)$.

a) benzene

\begin{tabular}{c|c|c|c|c|c|c}
\hline $\begin{array}{c}T \\
{[\mathrm{~K}]}\end{array}$ & $n_{\mathrm{a}}$ & $n_{\mathrm{i}}$ & $\begin{array}{c}\rho^{\prime} \\
{\left[\mathrm{kg} \cdot \mathrm{m}^{-3}\right]}\end{array}$ & $B_{\text {rel }}$ & $\begin{array}{c}B_{0} \times 10^{16} \\
{\left[\mathrm{~m} \cdot \mathrm{V}^{-2}\right]}\end{array}$ & $\begin{array}{c}B_{\mathrm{m}} \times 10^{27} \\
{\left[\mathrm{~m}^{5} \cdot \mathrm{C}^{2} \cdot \mathrm{J}^{-2}\right]}\end{array}$ \\
\hline 293 & 1.5147 & 1.4893 & 874.0 & 1.0 & 70 & 76 \\
298 & 1.5115 & 1.4862 & 873.2 & 0.96 & 67 & 74 \\
304 & 1.5077 & 1.4824 & 866.7 & 0.92 & 64 & 72 \\
313 & 1.5019 & 1.4767 & 857.1 & 0.89 & 63 & 71 \\
320 & 1.4975 & 1.4723 & 849.6 & 0.87 & 61 & 71 \\
328 & 1.4923 & 1.4673 & 840.9 & 0.81 & 57 & 68 \\
333 & 1.4891 & 1.4641 & 835.6 & 0.79 & 55 & 67
\end{tabular}


b) toluene

\begin{tabular}{c|c|c|c|c|c|c}
\hline$T$ & $n_{\mathrm{a}}$ & $n_{\mathrm{i}}$ & $\rho$ & $B_{\text {rel }}$ & $B_{0} \times 10^{16}$ & $B_{\mathrm{m}} \times 10^{27}$ \\
\hline 293 & 1.5100 & 1.4857 & 865.7 & 1.40 & 98 & 128 \\
299 & 1.5068 & 1.4827 & 860.1 & 1.37 & 96 & 127 \\
306 & 1.5030 & 1.4792 & 853.5 & 1.35 & 95 & 127 \\
313 & 1.4992 & 1.4757 & 846.9 & 1.33 & 93 & 128 \\
320 & 1.4954 & 1.4722 & 840.4 & 1.30 & 91 & 127 \\
328 & 1.4911 & 1.4682 & 832.8 & 1.30 & 91 & 127 \\
336 & 1.4868 & 1.4642 & 825.3 & 1.25 & 88 & 126 \\
343 & 1.4830 & 1.4607 & 818.8 & 1.20 & 84 & 123 \\
349 & 1.4798 & 1.4577 & 813.1 & 1.15 & 81 & 119 \\
355 & 1.4765 & 1.4547 & 807.5 & 1.10 & 77 & 116 \\
363 & 1.4722 & 1.4507 & 800.0 & 1.08 & 76 & 116 \\
370 & 1.4784 & 1.4472 & 793.4 & 0.80 & 56 & 88
\end{tabular}

c) nitrobenzene

\begin{tabular}{l|l|l|l|l|l|l}
\hline 293.6 & 1.5743 & 1.5340 & 1198 & 4.18 & 293 & 329 \\
301.9 & 1.5700 & 1.5293 & 1190 & 4.02 & 282 & 322 \\
311.3 & 1.5651 & 1.5236 & 1184 & 3.84 & 269 & 313 \\
323 & 1.5591 & 1.5173 & 1174 & 3.61 & 253 & 300 \\
335 & 1.5529 & 1.5104 & 1165 & 3.38 & 237 & 287 \\
358 & 1.5411 & 1.4973 & 1147 & 2.94 & 206 & 261 \\
373 & 1.5334 & 1.4888 & 1135 & 2.65 & 186 & 242 \\
393 & 1.5231 & 1.4774 & 1120 & 2.26 & 159 & 215 \\
423 & 1.5077 & 1.4604 & 1096 & 1.68 & 118 & 169 \\
434.5 & 1.5018 & 1.4538 & 1087 & 1.46 & 102 & 150
\end{tabular}

d) chlorobenzene

\begin{tabular}{l|l|l|l|l|l|l}
\hline 295.5 & 1.5372 & 1.5111 & 1103 & 2.36 & 166 & 196 \\
303 & 1.5332 & 1.5074 & 1095 & 2.25 & 158 & 190 \\
315 & 1.5269 & 1.5014 & 1083 & 2.14 & 154 & 190 \\
323.5 & 1.5224 & 1.4971 & 1073 & 2.08 & 146 & 183 \\
341 & 1.5131 & 1.4884 & 1055 & 1.87 & 132 & 172 \\
358 & 1.5041 & 1.4799 & 1037 & 1.77 & 124 & 167 \\
376 & 1.4946 & 1.4709 & 1017 & 1.58 & $111^{\star}$ & 156
\end{tabular}


e) 2-bromoanizole

\begin{tabular}{l|c|c|c|c|c|c}
\hline$T$ & $n_{\mathrm{a}}$ & $n_{\mathrm{i}}$ & $\rho$ & $B_{\text {rel }}$ & $B_{0} \times 10^{16}$ & $B_{\mathrm{m}} \times 10^{27}$ \\
\hline 298 & 1.5876 & 1.5566 & 1494 & 2.45 & 172 & 226 \\
313 & 1.5807 & 1.5494 & 1468 & 2.30 & 161 & 219 \\
326 & 1.5747 & 1.5432 & 1446 & 2.15 & 151 & 210 \\
346 & 1.5671 & 1.5336 & 1411 & 2.05 & 144 & 210 \\
367 & 1.5559 & 1.5235 & 1376 & 1.96 & 138 & 210 \\
387 & 1.5467 & 1.5139 & 1341 & 1.86 & 131 & 209 \\
404 & 1.5388 & 1.5057 & 1312 & 1.76 & 124 & 206 \\
424 & 1.5296 & 1.4961 & 1278 & 1.64 & 115 & 179 \\
441 & 1.5218 & 1.4880 & 1249 & 1.40 & 98 & 134
\end{tabular}

f) 2-methylbenzaldehyde

\begin{tabular}{l|l|l|l|l|l|l}
\hline 292 & 1.5668 & 1.5320 & 1037 & 3.00 & 211 & 269 \\
304.2 & 1.5606 & 1.5260 & 1027 & 2.91 & 204 & 267 \\
315.2 & 1.5551 & 1.5208 & 1019 & 2.68 & 188 & 251 \\
325 & 1.5500 & 1.5159 & 1011 & 2.48 & 174 & 236 \\
335 & 1.5449 & 1.5111 & 1004 & 2.41 & 169 & 234 \\
345 & 1.5400 & 1.5062 & 996 & 2.95 & 165 & 229 \\
354 & 1.5353 & 1.5019 & 989 & 2.25 & 158 & 226 \\
364 & 1.5303 & 1.4970 & 982 & 2.16 & 152 & 220 \\
373 & 1.5372 & 1.4926 & 982 & 2.09 & 147 & 214 \\
392 & 1.5275 & 1.4834 & 960 & 1.97 & 139 & 178 \\
411.5 & 1.5177 & 1.4739 & 943 & 1.87 & 131 & 207 \\
426 & 1.5078 & 1.4645 & 930 & 1.69 & 118 & 194
\end{tabular}

g) 3-methylbenzaldehyde

\begin{tabular}{l|l|l|l|l|l|l}
\hline 295 & 1.5940 & 1.5472 & 1022 & 3.13 & 220 & 273 \\
302 & 1.5925 & 1.5461 & 1016 & 2.64 & 185 & 232 \\
315 & 1.5900 & 1.5442 & 1006 & 2.23 & 161 & 199 \\
327 & 1.5875 & 1.5423 & 997.2 & 2.29 & 156 & 207 \\
339 & 1.5851 & 1.5404 & 988.0 & 2.09 & 147 & 191 \\
359 & 1.5826 & 1.5386 & 979.7 & 2.08 & 146 & 193 \\
361 & 1.5804 & 1.5369 & 971.3 & 1.92 & 135 & 180 \\
369 & 1.5789 & 1.5356 & 965.2 & 1.70 & 119 & 161 \\
379 & 1.5767 & 1.5340 & 957.6 & 1.55 & 109 & 149 \\
390 & 1.5745 & 1.5323 & 949.3 & 1.63 & 114 & 159
\end{tabular}


g) 3-methylbenzaldehyde (cont.)

\begin{tabular}{l|c|c|c|c|c|c}
\hline$T$ & $n_{\mathrm{a}}$ & $n_{\mathrm{i}}$ & $\rho$ & $B_{\text {rel }}$ & $B_{0} \times 10^{16}$ & $B_{\mathrm{m}} \times 10^{27}$ \\
\hline 400 & 1.5723 & 1.5307 & 941.7 & 1.62 & 114 & 159 \\
410 & 1.5703 & 1.5292 & 934.1 & 1.28 & 90 & 127 \\
422 & 1.5678 & 1.5273 & 925.0 & 1.42 & 100 & 143 \\
433 & 1.5910 & 1.5256 & 916.6 & 1.49 & 105 & 150 \\
443 & 1.5635 & 1.5223 & 909.0 & 1.49 & 105 & 154 \\
454 & 1.5612 & 1.5223 & 900.6 & 1.41 & 99 & 149
\end{tabular}

h) 3-chlorobenzaldehyde

\begin{tabular}{l|l|l|l|l|l|l}
\hline 296 & 1.5871 & 1.5473 & 1237 & 4.15 & 291 & 354 \\
316 & 1.5743 & 1.5376 & 1207 & 3.83 & 269 & 331 \\
338 & 1.5649 & 1.5269 & 1174 & 3.65 & 256 & 341 \\
359.5 & 1.5558 & 1.5166 & 1141 & 3.54 & 248 & 347 \\
381 & 1.5466 & 1.5060 & 1109 & 3.22 & 226 & 332 \\
402 & 1.5377 & 1.4958 & 1078 & 2.97 & 208 & 322 \\
423.5 & 1.5285 & 1.4853 & 1045 & 2.97 & 208 & 340
\end{tabular}

i) cinnamaldehyde

\begin{tabular}{l|l|l|l|l|l|l}
\hline 295 & 1.6596 & 1.5839 & 1048 & 6.31 & 443 & 532 \\
314 & 1.6488 & 1.5801 & 1032 & 5.25 & 368 & 456 \\
333 & 1.6380 & 1.5717 & 1016 & 5.16 & 362 & 464 \\
352 & 1.6272 & 1.5634 & 1000 & 4.85 & 340 & 452 \\
371 & 1.6164 & 1.5550 & 984.5 & 4.32 & 303 & 418 \\
390 & 1.6056 & 1.5467 & 968.6 & 4.11 & 279 & 412 \\
405 & 1.5970 & 1.5401 & 956.1 & 3.95 & 277 & 408 \\
423 & 1.5868 & 1.5436 & 941.0 & 3.57 & 265 & 397 \\
438 & 1.5783 & 1.5387 & 928.4 & 3.57 & 250 & 385 \\
451 & 1.5709 & 1.5345 & 917.6 & 3.41 & 240 & 377 \\
191 & 1.5635 & 1.5303 & 906.7 & 2.94 & 207 & 333
\end{tabular}

With the molar Kerr constants versus temperature available, we were in a position to calculate the contributions $B_{\mathrm{m}}^{\mathrm{NL}}$ and $B_{\mathrm{m}}^{\mathrm{RT}}$ from $\mathrm{Eq} .(6)$ and, hence, the values of $c$ and $\Gamma^{2}$. The results of these calculations for room temperature are shown in Table II together with the \% contribution of Voigt's effect to the optically induced birefringence. 
TABLE II The nonlinear molar Kerr constants, nonlinear polarizabilities and squared effective optical anisotropies determined experimentally for the liquids studied, at $293 \mathrm{~K}\left(\Delta B_{\mathrm{m}}^{\mathrm{NL}}=\Delta C= \pm 50 \%, \Delta \Gamma^{2}= \pm 20 \%\right)$.

\begin{tabular}{l|c|c|c|c}
\hline \hline Liquid & $\begin{array}{r}B_{\mathrm{m}}^{\mathrm{NL}} \times 10^{27} \\
{\left[\mathrm{~m}^{5} \cdot \mathrm{C}^{2} \cdot \mathrm{J}^{-2}\right]}\end{array}$ & $\begin{array}{r}c \times 10^{61} \\
{\left[\mathrm{C}^{2} \cdot \mathrm{m}^{4} \cdot \mathrm{J}^{-3}\right]}\end{array}$ & $\begin{array}{r}\Gamma^{2} \times 10^{80} \\
{\left[\mathrm{C}^{4} \cdot \mathrm{m}^{4} \cdot \mathrm{J}^{-2}\right]}\end{array}$ & $\begin{array}{r}B_{\mathrm{m}}^{\mathrm{NL}} / B_{\mathrm{m}} \\
{[\%]}\end{array}$ \\
\hline benzene & 6 & 7.6 & 18.6 & 7.6 \\
\hline toluene & 14 & 18.9 & 32.0 & 11.1 \\
\hline nitrobenzene & 13 & 17.1 & 84.7 & 4.0 \\
\hline chlorobenzene & 14 & 18.3 & 49.4 & 7.0 \\
\hline 2-bromoanisole & 109 & 142.6 & 29.3 & 47.7 \\
\hline 2-methylbenzaldehyde & 37 & 49.7 & 96.6 & 13.9 \\
\hline 3-methylbenzaldehyde & 25 & 32.0 & 49.0 & 8.9 \\
\hline 3-chlorobenzaldehyde & 37 & 48.5 & 90.3 & 10.4 \\
\hline cinnamaldehyde & 95 & 125.7 & 112.1 & 17.8
\end{tabular}

\section{Conclusions}

We report the results of a systematic study of the temperature dependence of optical Kerr effect in benzene and eight selected molecular liquids. The results obtained for benzene are in good agreement with available literature data [6] which proves reliability of our equipment. Our study describes a new method for determination of mean nonlinear polarizability of the molecules as well as the square of their effective optical anisotropy in molecular liquids as proposed in Ref. [10]. However, the method involves the neglect of the influence of molecular interactions on the Voigt's effect. Their influence is taken into account in the Langevin effect only (by way of the optical anisotropy). This limitation complicates the analysis of the temperature-dependent results.

Another important limitation of this method results from the employed extrapolation of the temperature dependence of the optical Kerr constant to zero temperature, which requires to assume that this dependence is linear also below room temperature. As follows from our studies [11], this assumption is not always correct. Therefore, the results obtained according to this method seem to be more of qualitative nature. Moreover, one should bear in mind that in higher temperatures the distribution of molecules becomes increasingly chaotic, especially as one approaches the boiling point of the liquid, and the influence of molecular interactions on the optical properties of the medium decreases. Hence, as the temperature varies, so do the contributions from different molecular configurations to the effective optical anisotropy. This can explain the differences in shape of the Kerr constant $B_{\text {rel }}$ versus temperature among the liquids measured in the present work.

Despite the aforementioned limitations the method gives quite satisfactory 
results. For example the values of the mean nonlinear polarizability we obtained applying this method for benzene $(c=7.6[\mathrm{SI}])$ are in good agreement with the literature data ( $c=8 \pm 4$ [SI]) obtained by other methods [12].

The results given in Table I show that in all cases OKE decreases with growing temperature; albeit, this decrease differs slightly from one liquid to another. For benzene and 2-bromoanisole it is linear throughout the whole temperature range studied, whereas for the other three liquids it diverges from linearity starting from some tens of degrees upward of room temperature, where the effect decreases more steeply than with $T^{-1}$ as the temperature increases. This behaviour of the temperature dependence of $\mathrm{OKE}$ enables us to draw certain conclusions concerning the local structure of the liquids, since the deviations from the $T^{-1}$-dependence point to changes in the effective optical anisotropy of the molecules in the liquid and, thus, to changes in local structure. However, in order to obtain more detailed information, further temperature measurements of OKE in other liquids with similar molecules are necessary.

\section{Appendix}

Coefficients listed in Table A and B have been calculated as described in Sec. 3 .

TABLE A The value of coefficients $a_{n}$ and $b_{n}$ describing the temperature dependence of the refractivity of the liquids studied.

\begin{tabular}{l|c|c|c|c}
\hline \hline \multirow{2}{*}{\multicolumn{1}{c|}{ Liquid }} & \multicolumn{2}{|c|}{$\lambda_{\mathrm{a}}=488 \mathrm{~nm}$} & \multicolumn{2}{c}{$\lambda_{\mathrm{i}}=694.3 \mathrm{~nm}$} \\
\cline { 2 - 5 } & $a_{n}$ & $\begin{array}{c}b_{n} \times 10^{4} \\
{\left[\mathrm{~K}^{-1}\right]}\end{array}$ & $a_{n}$ & $\begin{array}{c}b_{n} \times 10^{4} \\
{\left[\mathrm{~K}^{-1}\right]}\end{array}$ \\
\hline benzene & 1.699 & -6.500 & 1.669 & -6.200 \\
\hline toluene & 1.668 & -5.400 & 1.632 & -5.000 \\
\hline nitrobenzene & 1.725 & -5.147 & 1.707 & -5.885 \\
\hline chlorobenzene & 1.694 & -5.292 & 1.659 & -4.999 \\
\hline 2-bromoanisole & 1.725 & -4.600 & 1.543 & -4.800 \\
\hline 2-methylbenzaldehyde & 1.714 & -5.031 & 1.673 & -4.815 \\
\hline 3-methylbenzaldehyde & 1.653 & -2.003 & 1.544 & -1.524 \\
\hline 3-chlorobenzaldehyde & 1.707 & -4.300 & 1.692 & -4.861 \\
\hline cinnamaldehyde & 1.828 & -5.690 & 1.679 & -3.211
\end{tabular}


TABLE B

The value of coefficients $a_{\rho}$ and $b_{\rho}$ describing the temperature dependence of the density of the liquids studied.

\begin{tabular}{l|c|c|c}
\hline \hline \multicolumn{1}{c|}{ Liquid } & $\begin{array}{c}\rho \\
{\left[\mathrm{kg} \cdot \mathrm{m}^{-3}\right]}\end{array}$ & $\begin{array}{c}a_{\rho} \\
{\left[\mathrm{kg} \cdot \mathrm{m}^{-3}\right]}\end{array}$ & $\begin{array}{c}b_{\rho} \times 10^{3} \\
{\left[\mathrm{~kg} \cdot \mathrm{m}^{-3} \cdot \mathrm{K}^{-1}\right]}\end{array}$ \\
\hline benzene & 875 & 1193 & -1072 \\
\hline toluene & 865 & 1141 & -939 \\
\hline nitrobenzene & 1196 & 1428 & -783 \\
\hline chlorobenzene & 1107 & 1419 & -1067 \\
\hline 2-bromoanisole & 1502 & 2003 & -1710 \\
\hline 2-methylbenzaldehyde & 1023 & 1259 & -763 \\
\hline 3-methylbenzaldehyde & 1023 & 1246 & -760 \\
\hline 3-chlorobenzaldehyde & 1241 & 1680 & -1500 \\
\hline cinnamaldehyde & 1048 & 1295 & -837
\end{tabular}

\section{Acknowledgements}

The authors wish to express their gratitude to Prof. F. Kaczmarek for kind interest and valuable discussions.

This study was carried out within the Project CPBR 8.14/1.

\section{References}

[1] Z. Błaszczak, Acta Phys. Pol. A71, 601 (1987).

[2] S. Kielich, Proc. Phys. Soc. London 90, 847 (1967).

[3] S. Woźniak, in: Spektroskopia wielofotonowa, Ed. S. Kielich, UAM Publishers, Poznañ 1981, p. 101 (in Polish).

[4] S. Kielich, S. Woźniak, Acta Phys. Pol. A39, 233 (1971).

[5] Z. Błaszczak, Acta Phys. Pol. A66, 657 (1984).

[6] F.B. Martin, J.R. Lalanne, Opt. Commun. 2, 219 (1970).

[7] Z. Błaszczak, Acta Phys. Pol. A68, 629 (1985).

[8] Z. Błaszczak, Opt. Commun. 58, 439 (1986).

[9] Z. Błaszczak, P. Gauden, J. Chem. Soc. Faraday Trans. II 8, 239 (1988).

[10] S. Kielich, J.R. Lalanne, F.B. Martin, J. Phys. (France) 33, C1-191 (1972).

[11] Z. Błaszczak, P. Gauden, in preparation.

[12] M.P. Bogaard, A.D. Buckingham, G.L.D. Ritchide, Mol. Phys. 18, 575 (1970). 